\title{
Monte Carlo renormalization: The triangular Ising model as a test case
}

\author{
Wenan Guo, ${ }^{1,2, *}$ Henk W. J. Blöte, ${ }^{3,4}$ and Zhiming Ren ${ }^{1}$ \\ ${ }^{1}$ Physics Department, Beijing Normal University, Beijing 100875, China \\ ${ }^{2}$ The Abdus Salam International Centre for Theoretical Physics, Trieste, Italy \\ ${ }^{3}$ Faculty of Applied Sciences, Delft University of Technology, P.O. Box 5046, 2600 GA Delft, The Netherlands \\ ${ }^{4}$ Lorentz, Institute, Leiden University, P.O. Box 9506, 2300 RA Leiden, The Netherlands
}

(Received 23 December 2004; published 18 April 2005)

\begin{abstract}
We test the performance of the Monte Carlo renormalization method in the context of the Ising model on a triangular lattice. We apply a block-spin transformation which allows for an adjustable parameter so that the transformation can be optimized. This optimization purportedly brings the fixed point of the transformation to a location where the corrections to scaling vanish. To this purpose we determine corrections to scaling of the triangular Ising model with nearest- and next-nearest-neighbor interactions by means of transfer-matrix calculations and finite-size scaling. We find that the leading correction to scaling just vanishes for the nearestneighbor model. However, the fixed point of the commonly used majority-rule block-spin transformation appears to lie well away from the nearest-neighbor critical point. This raises the question whether the majority rule is suitable as a renormalization transformation, because the standard assumptions of real-space renormalization imply that corrections to scaling vanish at the fixed point. We avoid this inconsistency by means of the optimized transformation which shifts the fixed point back to the vicinity of the nearest-neighbor critical Hamiltonian. The results of the optimized transformation in terms of the Ising critical exponents are more accurate than those obtained with the majority rule.
\end{abstract}

DOI: 10.1103/PhysRevE.71.046126

PACS number(s): 05.50.+q, 64.60.Cn, 64.60.Fr, 75.10.Hk

\section{INTRODUCTION}

The Monte Carlo renormalization group (MCRG) was first introduced by $\mathrm{Ma}$ [1] and developed into an efficient tool for the investigation of phase transitions by Swendsen $[2,3]$. Many applications have been reported. Especially the three-dimensional Ising model has been the subject of extensive research efforts [4-8]. The convergence of the method appears to depend on the number of renormalization steps and on the number and character of the couplings included in the renormalization analysis. It is natural that the results [4-8] have become more accurate following developments of the algorithms and computer technology. However, it proved difficult to compete with the accuracies obtained for the three-dimensional Ising model by means of other techniques; see, e.g., Ref. [9] and references therein. The MCRG method using the majority rule has been observed to display a rather irregular convergence with the number of renormalization steps [8]. To improve the convergence, Swendsen [10] had already suggested a modified blocking rule which approximately transforms the nearest-neighbor Ising model at its critical point into itself, which thus also assumes the role of a fixed point. Corrections to scaling are usually associated with irrelevant scaling fields, but irrelevant scaling fields do not necessarily lead to corrections to scaling. Here we recall the so-called redundant operators [11] which do not influence the critical singularity. Shankar and Gupta [12] identified redundant operators and their eigenvalues from the majority-rule MCRG data of two-dimensional (2D) Ising model on the square lattice. A redundant operator in the $3 \mathrm{D}$

\footnotetext{
*Electronic address: waguo@bnu.edu.cn
}

Ising model has also been reported by Baillie et al. [8], who used a majority-rule block-spin transformation. The existence of redundant operators may suggest the possibility to move the fixed point along the direction of a redundant field by means of a modification of the blocking rule. If, in this way, the fixed point could be shifted to the original simulated Hamiltonian, then immediate convergence to the fixed point would be realized [10]. However, Fisher and Randeria [13] pointed out that, in general, the thermodynamical observables of the simulated Hamiltonian will display unique corrections to scaling. According to the theory based on assumptions which include the existence of the transformation and its analyticity, such corrections are generated by irrelevant scaling fields. Thus, the corrections should vanish if one uses a transformation that brings the fixed point to the simulated Hamiltonian. It is clear that something is wrong or missing in this picture; the corrections to scaling of the original Hamiltonian are in general nonzero and should obviously be independent of the renormalization transformation used to analyze the system. Possible explanations stipulate that the assumptions made in real-space renormalization theory are invalid. For instance, the transformation between the original and the renormalized couplings may be ill defined [14]_-i.e., not exist in the thermodynamic limit. Or the assumption of analyticity of the transformation may be wrong. In that case nonanalytic contributions might enter in the "analytic" part of the transformation or in the renormalized couplings.

Reference [15] addressed the question of the analyticity of the block-spin transformation in the context of the threedimensional Ising model. Indeed it reported strong singular behavior, albeit for a somewhat unusual transformation, which involved only weak correlations between the block spin and the original spins. No strong nonanalyticities were found for "normal" transformations. That work also ad- 


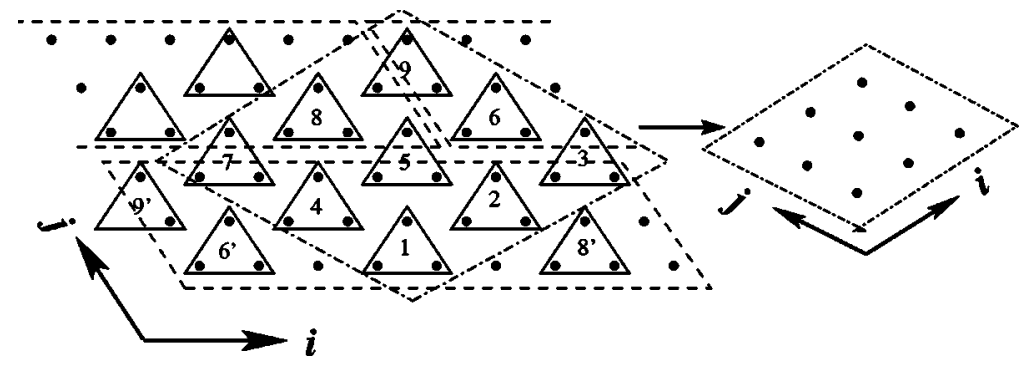

FIG. 1. Triangular lattice and blocking procedure. The dashed boxes contain periodic units of a $L \times L / 3$ lattice. The "shifted" stacking of these boxes serves to satisfy the hexagonal symmetry of the periodic boundary conditions of the system. In order to define the lattice as a two-dimensional array labeled with indices $i$ and $j$, the edges of these boxes are chosen along lattice edges. The blocking procedure combines three spins (black dots) into a block spin (triangle). The block spins (shown as black dots on the right) form another triangular lattice of size $L / 3 \times L / 3$, which fits in an ordinary rhombus-shaped periodic box.

dressed the question whether the transformation could be modified such that consistency is restored with the standard assumptions in real-space renormalization theory, which imply that the corrections to scaling vanish at the fixed point of the transformation. This question was investigated for a critical 3D Ising model with second- and third-neighbor interactions, chosen such as to ensure that the leading correction to scaling is strongly suppressed. The optimization of the block-spin transformation such that the fixed point is close to the simulated Hamiltonian then apparently resulted in fast convergence and a relatively high accuracy of the critical exponents.

In the present work we investigate the analogous problem in two dimensions. Whereas there seems little reason for accurate determinations of exactly known critical exponents, the question under what circumstances the MCRG technique works well is a fundamental and important issue, because it leads to insight into the properties of real-space renormalization transformations. While the corrections to scaling and their associated irrelevant fields may be more difficult to observe in two than in three dimensions, the choice of two dimensions alleviates restrictions due to the rapid increase of the computer time requirements with system size, as they apply to three-dimensional models. One of the reasons to select the triangular Ising model is that one can define a block-spin transformation such that each blocking step reduces the number of spins by factor of only 3 . Thus, a relatively large number of blocking steps can be performed.

First, we shall demonstrate that the leading correction to scaling vanishes at the nearest-neighbor Hamiltonian of this model. We show this by means of transfer-matrix calculations and finite-size scaling. Next, we determine the fixed point of the commonly used majority-rule block-spin transformation. We find that it is well separated from the nearestneighbor critical point. However, a modified block-spin transformation brings it back to the vicinity of the critical nearest-neighbor Hamiltonian. In analogy with the threedimensional case, we may expect that consistency with the assumption of analyticity will then yield better convergence and more accurate results. A test of this idea is facilitated because the critical point and exponents of this model are exactly known. Moreover, since we can perform relatively many blocking steps, we have a better chance to quantitatively determine the finite-size effect, as well as the renor- malization effect associated with the approach of the fixed point.

This paper is organized as follows. Section II briefly reviews the MCRG method and some further technical details. In Sec. III, we present the transfer-matrix analysis of the corrections to scaling as a function of the ratio of the nearestneighbor and next-nearest-neighbor interactions and determine the points where these corrections vanish. Then, we calculate the fixed point of the majority rule and introduce a modified transformation in order to bring the fixed point to the nearest-neighbor critical point. In Sec. IV, we compare the convergence and accuracy of the majority rule and the modified transformation using the results for the eigenvalues of the linearized transformation matrix. We discuss our findings in Sec. V.

\section{MODEL AND METHOD}

Since the MCRG method has amply been reviewed $[3,6]$, we present only a brief outline. The reduced Hamiltonian of the Ising model can be written compactly:

$$
H(S)=-\sum_{\alpha=0}^{\infty} K_{\alpha} S_{\alpha},
$$

where $S$ is a spin configuration, the $K_{\alpha}$ are couplings, and the $S_{\alpha}$ are the conjugate lattice sums of spin products. For example, $K_{1}$ may be the magnetic field and $S_{1}=\sum_{i} s_{i}$ the sum of all spins; $K_{2}$ may be the nearest-neighbor coupling and $S_{2}$ $=\sum_{\langle\mathrm{NN}\rangle} s_{i} s_{j}$ the sum over all nearest-neighbor spin products. A special "coupling" is the background energy density $K_{0}$ which is conjugate to the number of spins $S_{0}$.

Application of a block-spin transformation to Monte Carlo-generated configurations $S$ leads to configurations $S^{\prime}$ described by a Hamiltonian $H^{\prime}=H\left(K_{0}^{\prime}, K_{1}^{\prime}, K_{2}^{\prime}, \ldots ; S^{\prime}\right)$. The renormalized couplings $K_{\alpha}^{\prime}$ are assumed to be analytic functions of the original ones, even at the infinite-system critical point.

For the present renormalization analysis of the Ising model on the triangular lattice we follow the spin-blocking method introduced by Niemeijer and van Leeuwen [16]. As shown in Fig. 1, we divide the lattice into up triangles of three site each. Each such triangle is then replaced by a block spin $s^{\prime}$ with a probability $P\left(s^{\prime} ; s_{1}, s_{2}, s_{3}\right)$ where $s_{1}, s_{2}, s_{3}$ are 
the three original spins; this probability defines the renormalization transformation.

Application of this blocking procedure leads to a system of block spins that form another triangular lattice. In a finite system of block spins, careful consideration must be given to a technical problem concerning the choice of the axes in relation with the periodic boundary conditions. As shown in Fig. 1, the block spins form a lattice that is rotated by $\pi / 2$ (or $\pi / 6$ ) with respect to the original lattice. Let the original lattice have size $L \times L$, with periodic boundary conditions in both lattice directions-i.e., $s_{L+1, j}=s_{1, j}$ and $s_{i, L+1}=s_{i, 1}$. Let the vectors spanning the original elementary cell be $\vec{e}_{1}=\hat{x}$ and $\vec{e}_{2}=(\sqrt{3} \hat{y}-\hat{x}) / 2$ where $\hat{x}$ and $\hat{y}$ are Cartesian unit vectors. The vectors describing the size of the periodic box can simply be chosen as $L \vec{e}_{1}$ and $L \vec{e}_{2}$. The periodic box of the block-spin lattice has obviously the same size when expressed in original lattice units. But the rotation of the lattice and the larger size of the elementary cell prescribe the choice of a periodic box of a different shape, for instance, spanned by vectors $L\left(2 \vec{e}_{2}+\vec{e}_{1}\right)$ and $L \vec{e}_{2}$. This box has the same area as the original one, and the spanning vectors are integer linear combinations of the original ones; it is thus equivalent. The former vector has a length $L \sqrt{3}$, which covers precisely $L$ edges of the lattice of block spins, so that the vector defines a lattice axis along which the spins can be counted. However, the latter vector does not have this property. We use $-L\left(2 \vec{e}_{1}\right.$ $\left.+\vec{e}_{2}\right) / 3$ instead. It has a length of $L / 3$ new lattice units. The box spanned by $L\left(2 \vec{e}_{2}+\vec{e}_{1}\right),-L\left(2 \vec{e}_{1}+\vec{e}_{2}\right) / 3$ provides a natural way to label and store the $L / 3$ block spins by two coordinates $i=1, \ldots, L$ and $j=1, \ldots, L / 3$. Note that $L \vec{e}_{2}$ [and $n o t$ $\left.-L\left(2 \vec{e}_{1}+\vec{e}_{2}\right) / 3\right]$ still describes one direction of periodic shift in accordance with the periodic boundary conditions. Therefore, the boxes are periodically repeated in a "shifted" fashion, as shown in Fig. 1. The periodic unit composed of the block spins has the same hexagonal symmetry as the original system. After a second blocking operation, the lattice assumes the original orientation and one can, e.g., simply use the original periodic box spanned by $L \vec{e}_{1}, L \vec{e}_{2}$ which now accommodates $L / 3 \times L / 3$ sites. The mode of storage described here for the renormalized system can obviously also be used in the simulation of a system of $L^{2} / 3$ spins with boundary conditions of hexagonal symmetry.

The renormalization procedure can be iterated until the system becomes too small for useful analysis. We denote the renormalization level by superscripts. Thus, after $i$ renormalization transformations the Hamiltonian is $H^{(i)}$ $=H\left(K_{0}^{(i)}, K_{1}^{(i)}, K_{2}^{(i)}, \ldots ; S^{(i)}\right)$. The corresponding linearized renormalization transformation matrix

$$
T_{\alpha \beta}^{(i)} \equiv \frac{\partial K_{\alpha}^{(i)}}{\partial K_{\beta}^{(i-1)}}
$$

is related to lattice sum correlations via

$$
\sum_{\gamma>0} B_{\alpha \gamma}^{(i)} T_{\gamma \beta}^{(i)}=C_{\alpha \beta}^{(i)}
$$

where the correlations

$$
B_{\alpha \beta}^{(i)}=\left\langle\left\langle S_{\alpha}^{(i)} S_{\beta}^{(i)}\right\rangle\right\rangle=\left\langle S_{\alpha}^{(i)} S_{\beta}^{(i)}\right\rangle-\left\langle S_{\alpha}^{(i)}\right\rangle\left\langle S_{\beta}^{(i)}\right\rangle=\frac{\partial\left\langle S_{\alpha}^{(i)}\right\rangle}{\partial K_{\beta}^{(i)}}
$$

and

$$
C_{\alpha \beta}^{(i)}=\left\langle\left\langle S_{\alpha}^{(i)} S_{\beta}^{(i-1)}\right\rangle\right\rangle=\left\langle S_{\alpha}^{(i)} S_{\beta}^{(i-1)}\right\rangle-\left\langle S_{\alpha}^{(i)}\right\rangle\left\langle S_{\beta}^{(i-1)}\right\rangle=\frac{\partial\left\langle S_{\alpha}^{(i)}\right\rangle}{\partial K_{\beta}^{(i-1)}}
$$

can be calculated by means of Monte Carlo sampling; thus, also the $T_{\alpha \beta}^{(i)}$ can be calculated. Since the even and odd lattice sums are not correlated, this analysis can be performed separately in the even and odd coupling subspaces. Consideration has to be given to the boundary conditions which require different handling during the even and odd renormalization steps.

The fixed point of the transformation can also be found if the distance $\delta \vec{K}$ of the original Hamiltonian to the fixed point is small. For a triangular Ising system containing $3^{p}$ spins, the lattice sums calculated after $n$ renormalization steps on the remaining $3^{p-n}$ sites are denoted $S_{\alpha}^{(p, n)}$. One can linearize as

$$
\begin{aligned}
\left\langle S_{\alpha}^{(p+m, n+m)}\right\rangle-\left\langle S_{\alpha}^{(p, n)}\right\rangle= & \sum_{\beta}\left[\left\langle\left\langle S_{\alpha}^{(p+m, n+m)} S_{\beta}^{(p+m, 0)}\right\rangle\right\rangle\right. \\
& \left.-\left\langle\left\langle S_{\alpha}^{(p, n)} S_{\beta}^{(p, 0)}\right\rangle\right\rangle\right] \delta K_{\beta}
\end{aligned}
$$

and solve the distance $\delta \vec{K}$ from this equation.

The present MCRG calculations involve the following steps

(i) The generation of a critical spin configuration by means of the Metropolis and Wolff [17] methods. In this work, typically two Metropolis sweeps and ten Wolff cluster steps are used to generate a new spin configuration.

(ii) Calculation of the lattice sums $S_{\alpha}$.

(iii) Execution of the block-spin transformation which reduces the number of lattice sites by a factor of 3 .

(iv) The same as step (ii), using the reduced spin lattice.

(v) Repetition of steps (iii) and (iv). This sequence stops at system size $3 \times 3$.

(vi) Calculation and accumulation of the cross products $S_{\alpha}^{(i)} S_{\beta}^{(j)}$.

(vii) Repetition of steps (i)-(vi) (called a "cycle") for a large number of configurations.

The transformation matrix $T$ is approximated by solving Eq. (3) in a subspace spanned by a finite number of couplings. We have included up to ten even couplings and five odd couplings in our simulations, as defined in Fig. 2. As we shall see, this is enough to reach a satisfactory convergence of the eigenvalues. Under iteration of the block-spin transformation, the $K_{\alpha}^{(i)}(\alpha>0)$ are assumed to approach the fixed point of the transformation, where the eigenvalues of $T$ determine the critical exponents.

\section{CORRECTIONS TO SCALING AND THE FIXED POINT}

Models in the same universality class share the same type of leading singularities in thermodynamic quantities at their 


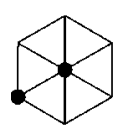

(1)

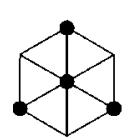

(6)

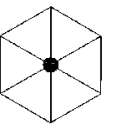

(1)

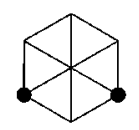

(2)

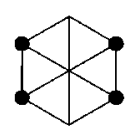

(7)

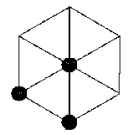

(2)

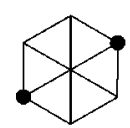

(3)

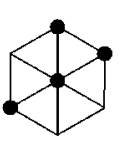

(8)

2

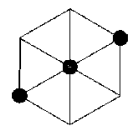

(3)

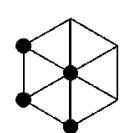

(4)

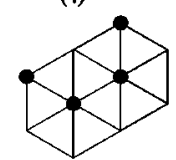

(9)

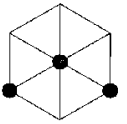

(4)

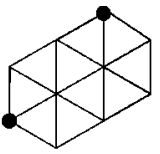

(5)

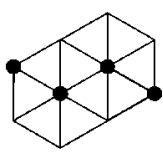

(10)

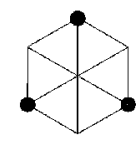

(5) b

FIG. 2. The even (a) and odd (b) couplings used in the present analysis. The black circles represent the spins participating in these couplings.

critical points. In general, also corrections to scaling are described by a common set of exponents within a universality class. We consider the free energy density of a model near criticality, as a function of a relevant temperature field $t$ and an irrelevant field $u$. According to the well-established framework of the renormalization theory, which assumes the existence of a critical fixed point and the analyticity of the transformation (see, e.g., Ref. [18]), its singular part has the following scaling behavior in the thermodynamic limit:

$$
\begin{aligned}
f_{s}(t, u) & =|t|^{d / y_{t}} f_{s}\left( \pm 1,|t|^{-y_{i} / y_{t}} u\right) \\
& =|t|^{d / y_{t}}\left[f_{s}( \pm 1,0)+a|t|^{-y_{i} / y_{t}} u+\cdots\right],
\end{aligned}
$$

where $y_{t}$ and $y_{i}$ are the relevant and irrelevant renormalization exponents, respectively. Differentiation of the free energy yields the scaling behavior of thermodynamic quantities. For instance, the specific heat behaves as

$$
C_{s}(t, u)=|t|^{d / y_{t}-2}\left[b_{0}+b_{1} u|t|^{-y_{i} / y_{t}}+\cdots\right] .
$$

The term with exponent $-y_{i} / y_{t}$-i.e., the correction to scaling - has an amplitude proportional to the irrelevant scaling field $u$, which is zero at the fixed point of the renormalization transformation. In general a point on a critical manifold displays nonzero corrections to scaling and can therefore not serve as a fixed point of a transformation satisfying the aforementioned assumptions.

Consider an Ising model with nearest-neighbor coupling $K_{N N}$ and next-nearest-neighbor coupling $K_{N N N}$. The irrelevant scaling field can be estimated by transfer-matrix calculations and finite-size scaling. Consider a triangular Ising model with nearest-neighbor coupling $K_{N N}$ and next-nearestneighbor coupling $K_{N N N}$. Instead of $\left(K_{N N}, K_{N N N}\right)$ we use the scaling fields $(t, u)$ to express the scaling properties. The model is wrapped on an infinitely long cylinder with a circumference of $L$ lattice edges. The transfer-matrix technique
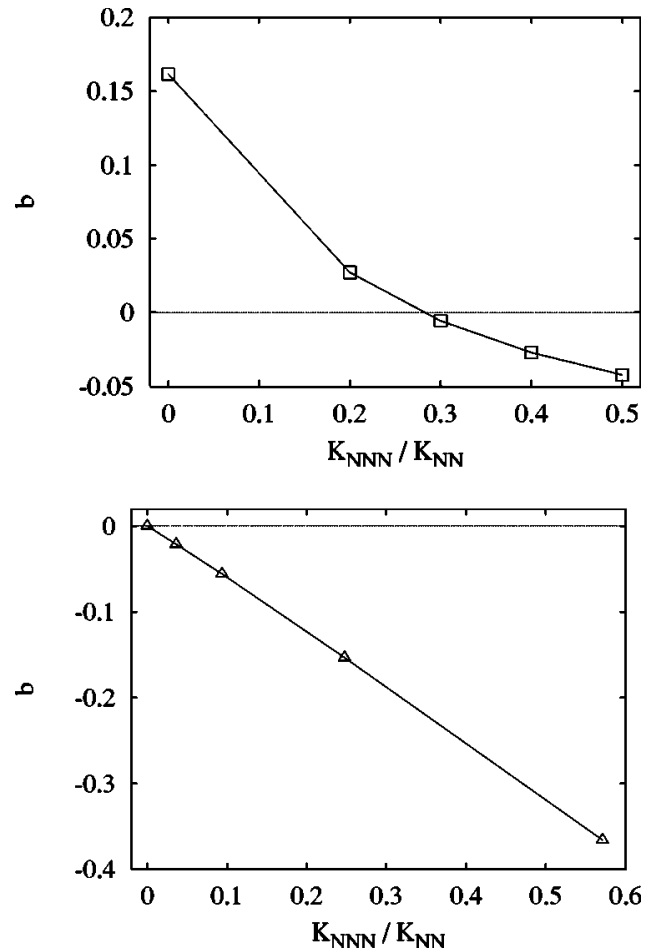

FIG. 3. Amplitude of the finite-size correction in the critical correlation length vs the coupling ratio $K_{N N N} / K_{N N}$. This amplitude is proportional to the irrelevant scaling field. (a) Ising model on the square lattice and (b) Ising model on the triangular lattice.

[19] involves the calculation of the correlation length $\xi$ from which the so-called scaled magnetic gap $X_{h}(L, t, u)$ $=L /\left[2 \pi \xi_{h}(L, t, u)\right]$ can be obtained. Expansion of its scaling equation leads to

$$
X_{h}(L, t, u)=X_{h}+a_{1} t L^{y_{t}}+a_{2} u L^{y_{i}}+\cdots .
$$

The magnetic scaling dimension satisfies $X_{h}=1 / 8$, and one has $y_{t}=1$ and $y_{i}=-2$ for the Ising model. For a given $K_{N N}$, one may express the equation

$$
X_{h}(L, t, u)=X_{h}
$$

in terms of $K_{N N}$ and $K_{N N N}$ and solve numerically for $K_{N N N}$ for a range of system sizes $L$. In the solutions, which are denoted $K_{N N N}(L)$, the effect of the irrelevant field, which is proportional to $u$, is compensated by a nonzero temperature field that asymptotically satisfies $t \propto u L^{-3}$-i.e., $K_{N N N}(L) \simeq K_{N N N, c}$ $+b L^{-3}$. Numerical analysis thus yields the amplitude $b$, which is proportional to the irrelevant scaling field, as well as the critical value of the next-nearest-neighbor coupling $K_{N N N, c}$, by means of extrapolation of the finite-size solutions $K_{N N N}(L)$.

Figure 3 shows the correction-to-scaling amplitudes $b$ versus the critical ratio $K_{N N N} / K_{N N}$ for the Ising models with ferromagnetic couplings on square and triangular lattices. Apparently the correction to scaling just vanishes for the nearest-neighbor Ising model on the triangular lattice, while it is appreciable for the nearest-neighbor Ising model on the square lattice. In the latter case, the leading correction to scaling vanishes at $K_{N N N} / K_{N N} \approx 0.3$. The result for the trian- 
gular lattice is in accordance with the existence of a fixed point in an exact renormalization analysis of the nearestneighbor triangular Ising model by Hilhorst et al. [20].

There seems to exist considerable freedom in the choice of the probability $P$ used in the block-spin transformation. For instance, one may adopt the majority rule, but there exist other possibilities, which we shall explore below. It has been a widespread belief is that the assumptions, on which the renormalization theory is based, are satisfied for a wide range of block-spin transformations. Thus, modification of the transformation rule was believed to shift the fixed point only along redundant directions, which do not affect the corrections to scaling. However, this belief was not supported by results reported in Ref. [15]. These results indicated instead that the requirement that the corrections to scaling vanish at the fixed point does impose a constraint on the transformation in the case of the three-dimensional Ising model.

A commonly chosen transformation is the so called majority-rule transformation: i.e.,

$$
P\left(s^{\prime} ; s_{1}, s_{2}, s_{3}\right)= \begin{cases}1 & \text { if } s^{\prime}=\operatorname{sgn}\left(s_{1}+s_{2}+s_{3}\right), \\ 0 & \text { if } s^{\prime} \neq \operatorname{sgn}\left(s_{1}+s_{2}+s_{3}\right) .\end{cases}
$$

The question arises naturally where is the fixed point of this transformation for the Ising model on the triangular lattice. According to Eq. (6), one can locate the fixed point of a transformation from a comparison of lattice sums of two systems with equal size but at different renormalization levels. These sums are thus obtained from simulations of two systems with different sizes. In order to determine the irrelevant scaling fields of the original Hamiltonian, the best results are expected for $n$ as small as possible. Thus we used $n=0, m$ $=1$ and solved $\delta K_{\alpha}$ on the basis of simulations of two subsequent system sizes in the even coupling subspaces of dimension 2 and 3 respectively. To reduce the linearization error, we have estimated the fixed point iteratively; i.e., we first simulated the nearest-neighbor Hamiltonian, estimated the fixed point Hamiltonian by solving Eq. (6). The latter Hamiltonian was then used for new simulations (which consisted only of Metropolis steps, because the presence of additional antiferromagnetic interactions affects the efficiency of cluster algorithms) and for a second solution of Eq. (6), etc. Although the estimated fixed points display a substantial finite-size dependence, apparent convergence is still found in both the two- and three-dimensional even coupling subspaces. The final results are shown in Tables I and II. Extrapolation of these finite-size data to infinity yields the location of the fixed point as listed in the last rows of the two tables.

Our final estimations show that the fixed point of the majority-rule transformation of the Ising model on the triangular lattice is well separated from the nearest-neighbor critical model. On the basis of the transfer-matrix results given above, we expect corrections to scaling with a positive amplitude $b$ at the fixed point, apparently in conflict with the underlying assumptions.

In order to restore consistency, we introduce a modified block-spin transformation such that the fixed point moves to the critical point of nearest-neighbor model. Following the
TABLE I. Fixed point of the majority rule, estimated in the two-dimensional even coupling subspace: finite-size data and extrapolated values. The errors in the last decimal places, shown in parentheses, were estimated from the variations in the results during the last iteration steps.

\begin{tabular}{cccc}
\hline \hline$L$ & $S$ & $K_{N N}^{*}$ & $K_{N N N}^{*}$ \\
\hline $9 \times 9$ & $9 \times 3$ & $0.3611(2)$ & $-0.0543(2)$ \\
$27 \times 9$ & $9 \times 9$ & $0.3518(2)$ & $-0.0488(2)$ \\
$27 \times 27$ & $27 \times 9$ & $0.3485(2)$ & $-0.0466(2)$ \\
$81 \times 27$ & $27 \times 27$ & $0.3468(2)$ & $-0.0455(2)$ \\
$81 \times 81$ & $81 \times 27$ & $0.3464(2)$ & $-0.0452(2)$ \\
$\infty$ & & $0.346(2)$ & $-0.045(1)$ \\
\hline \hline
\end{tabular}

lines of Ref. [15] the probability of a block spin $s^{\prime}$ is chosen as

$$
P\left(s^{\prime}\right)=\frac{\exp \left(\omega s^{\prime} s_{b}\right)}{2 \cosh \left(\omega s_{b}\right)}
$$

where $s_{b}$ is the sum of the spins on a triangular face: $s_{b}=s_{1}$ $+s_{2}+s_{3}$.

By varying $\omega$, it appears possible to adjust the solution $\delta K_{\alpha}$ of Eq. (6) in the two-dimensional even subspace (spanned by $K_{N N}$ and $K_{N N N}$ ) to a sufficiently small value. Our numerical results show that the value of $\omega$ has only a weak finite-size dependence. The best estimate is $\omega \approx 1.258$. With this value the block-spin transformation, truncated to the $n_{c}$ $=2$ subspace, does not significantly move the Hamiltonian away from the nearest-neighbor critical point.

\section{EIGENVALUES AND EXPONENTS}

To compare the two different block-spin rules, we have performed extensive MCRG simulations. Each simulation involved the generation of $10^{8}$ configurations, each of which was followed by the renormalization blocking and correlation procedures. Statistical errors of the lattice sums and correlations of the lattice sums are estimated by dividing the simulation a number of shorter runs and calculating the standard deviation between the results of the subruns.

TABLE II. Fixed point of the majority rule, estimated in the three-dimensional even coupling subspace: finite-size data and extrapolated values. The errors in the last decimal places, shown in parentheses, were estimated from the variations in the results during the last iteration steps.

\begin{tabular}{ccccc}
\hline \hline$L$ & $S$ & $K_{N N}^{*}$ & $K_{N N N}^{*}$ & $K_{3 N}^{*}$ \\
\hline $9 \times 9$ & $9 \times 3$ & $0.427(1)$ & $-0.0463(5)$ & $-0.0389(5)$ \\
$27 \times 9$ & $9 \times 9$ & $0.388(1)$ & $-0.0325(5)$ & $-0.0326(5)$ \\
$27 \times 27$ & $27 \times 9$ & $0.379(1)$ & $-0.0291(5)$ & $-0.0312(5)$ \\
$81 \times 27$ & $27 \times 27$ & $0.373(1)$ & $-0.0269(5)$ & $-0.0301(5)$ \\
$81 \times 81$ & $81 \times 27$ & $0.371(1)$ & $-0.0263(5)$ & $-0.0298(5)$ \\
$\infty$ & & $0.370(2)$ & $-0.026(1)$ & $-0.030(1)$ \\
\hline \hline
\end{tabular}


TABLE III. Largest eigenvalues of the majority-rule transformation matrix in the $n_{c}=5$ even coupling subspace. The statistical error in the data is approximately 0.0003 .

\begin{tabular}{cccccccc}
\hline \hline$i \backslash p$ & 10 & 9 & 8 & 7 & 6 & 5 & 4 \\
\hline 1 & 1.653704 & 1.653505 & 1.653410 & 1.653081 & 1.652479 & 1.650893 & 1.645550 \\
2 & 1.711253 & 1.711037 & 1.710827 & 1.710254 & 1.708959 & 1.703025 & 1.759714 \\
3 & 1.724861 & 1.724722 & 1.723996 & 1.722573 & 1.716360 & 1.777823 & \\
4 & 1.728788 & 1.728342 & 1.726711 & 1.720288 & 1.784270 & & \\
5 & 1.729631 & 1.728416 & 1.722031 & 1.786435 & & & \\
6 & 1.728706 & 1.722093 & 1.787168 & & & & \\
7 & 1.722542 & 1.787104 & & & & & \\
8 & 1.787676 & & & & & & \\
\hline \hline
\end{tabular}

According to the theory, a critical Hamiltonian parametrized by irrelevant fields $u_{1}$ and $u_{2}$ renormalizes towards the critical fixed point. After one step with scale factor $b$ $=\sqrt{3}$ it arrives at $u_{1} b^{y_{i}}, u_{2} b^{y_{j}}$, where $y_{i}$ and $y_{j}$ are the two leading irrelevant renormalization exponents. The leading eigenvalue in the even subspace of $T_{\alpha \beta}^{(i)}$, which is denoted $\lambda_{e}^{(i)}$, and that in the odd subspace, denoted $\lambda_{0}^{(i)}$, should thus approach the fixed-point values $\sqrt{3}$ and $(\sqrt{3})^{o 15 / 8}$, as dictated by the temperature and magnetic renormalization exponents $y_{t}$ $=1$ and $y_{h}=15 / 8$, respectively.

In practice, one is naturally subject to restrictions concerning the finiteness of the system and the truncation of the coupling space to a finite number of dimensions. We have included up to ten even couplings and five odd couplings in our analyses, both for the majority rule and the modified rule. We shall see that this is enough for the calculations presented in this paper. The largest system included in our analyses has $3^{10}$ sites-i.e., a $243 \times 243$ system. The smallest system that was simulated has $3^{3}$ sites-i.e., a $9 \times 3$ system.

The solution of Eq. (3) in terms of the linearized renormalization transformation matrix $T_{\alpha \beta}^{(i)}$ at the $i$ th renormalization level is still dependent on the level $i$ and the number $n_{c}$ of couplings included. Thus an extrapolation procedure has to be applied to the eigenvalues of the $T_{\alpha \beta}^{(i)}$. After $i$ renormalization steps of a system of $3^{p}$ sites the system has shrunk to $3^{p-i}$ sites. We denote the pertinent leading eigenvalues of $T_{\alpha \beta}^{(i)}$ truncated to $n_{c}$ dimensions as $\lambda_{e}^{\left(i, n_{c}\right)}(p)$ and $\lambda_{o}^{\left(i, n_{c}\right)}(p)$.

The renormalization effect on these eigenvalues, associated with the approach of the fixed point with the number of blocking steps, is described by standard renormalization theory as formulated above. However, a quantitative description of the finite-size effect of the renormalization transformation itself is not available. This effect may be associated with the truncation of the space of operators and has been found to be smooth and to decay fast [22]. These results agree with the assumption that renormalization transformations are local in character, but are restricted to relatively small finite sizes. Under these circumstances we attempt to describe the finite-size effect in terms of an expansion in the inverse number of sites.

Taking into account all these effects, one may extrapolate the leading eigenvalues at the fixed point according to the following fit formula:

$$
\begin{aligned}
\lambda_{e, o}^{\left(i, n_{c}\right)}(p)= & \lambda_{e, o}+b_{1}(\sqrt{3})^{i y_{i}}+b_{2}(\sqrt{3})^{i y_{j}}+c_{1} 3^{(p-i) y_{1}}+c_{2} 3^{(p-i) y_{2}} \\
& +c_{3} 3^{(p-i) y_{3}}+c_{4} 3^{(p-i) y_{4}}+d(\sqrt{3})^{i y_{i}} 3^{(p-i) y_{1}}
\end{aligned}
$$

where different sets of coefficients apply to the eigenvalues in the even and odd coupling subspaces. The exponents $y_{1}$, $y_{2}, y_{3}, y_{4}$ serve to describe the finite-size effect. They are set as $-1,-2,-3,-4$, respectively. The leading and subleading irrelevant renormalization exponents are denoted $y_{i}$ and $y_{j}$. The last term of this fit formula describes the mixed effect of renormalization and finite size.

\section{A. Results of the majority rule and the modified rule}

We first analyze the largest eigenvalues of the linearized transformation in the five-dimensional even and odd sub-

TABLE IV. Largest eigenvalues of the majority-rule transformation matrix in the $n_{c}=5$ odd coupling subspace. The statistical error in the data is approximately 0.00015 .

\begin{tabular}{cccccccc}
\hline \hline$i \backslash p$ & 10 & 9 & 8 & 7 & 6 & 5 & 4 \\
\hline 1 & 2.730199 & 2.730195 & 2.730211 & 2.730201 & 2.730195 & 2.730163 & 2.730076 \\
2 & 2.773272 & 2.773272 & 2.773260 & 2.773260 & 2.773204 & 2.773176 & 2.768895 \\
3 & 2.791232 & 2.791231 & 2.791204 & 2.791194 & 2.791069 & 2.786765 & \\
4 & 2.797330 & 2.797324 & 2.797274 & 2.797198 & 2.792882 & & \\
5 & 2.799578 & 2.799540 & 2.799433 & 2.795107 & & & \\
6 & 2.800388 & 2.800293 & 2.795968 & & & & \\
7 & 2.800598 & 2.796270 & & & & & \\
8 & 2.796371 & & & & & & \\
\hline \hline
\end{tabular}


TABLE V. Eigenvalues of the modified rule transformation matrix $(\omega=1.258)$ in the $n_{c}=5$ even coupling subspace. The estimated statistical error in the data is approximately 0.0003 .

\begin{tabular}{cccccccc}
\hline \hline$i \backslash p$ & 10 & 9 & 8 & 7 & 6 & 5 & 4 \\
\hline 1 & 1.708660 & 1.708615 & 1.708684 & 1.708735 & 1.708734 & 1.708597 & 1.709245 \\
2 & 1.725449 & 1.725540 & 1.725570 & 1.725460 & 1.725277 & 1.725550 & 1.710963 \\
3 & 1.729391 & 1.729291 & 1.729405 & 1.729316 & 1.729749 & 1.715674 & \\
4 & 1.731048 & 1.730904 & 1.730914 & 1.731122 & 1.717573 & & \\
5 & 1.731546 & 1.731354 & 1.732069 & 1.718616 & & & \\
6 & 1.731564 & 1.732176 & 1.718922 & & & & \\
7 & 1.732312 & 1.719175 & & & & & \\
8 & 1.719252 & & & & & & \\
\hline \hline
\end{tabular}

spaces by fitting the data with formula (12). This yields the extrapolated fixed-point values and allows a comparison between the results obtained by the two different transformations. We begin with the results obtained by the majority rule.

Table III lists the eigenvalues $\lambda_{e}^{(i, 5)}(p)$ of the majority-rule transformation matrix in the five-dimensional even coupling subspace. Preliminary fits show that the amplitudes of the terms with $3^{(p-i) y_{3}}$ and $3^{(p-i) y_{4}}$ are not significant. Since this holds for all fits including these terms, we neglected them. We set $y_{i}$ to be free in the fitting procedure, while $y_{j}$ is set as -4 . The fit yields the extrapolated eigenvalue as $\lambda_{e}$ $=1.7330(2)$ and the irrelevant exponent as $y_{i}=-1.69(5)$, different from the expected value -2 .

The statistical error of the raw data is estimated as 0.0003 . Then, this fit has a residual $\chi^{2}=833$, which is much too large in comparison with the number of degrees of freedom which is 29. Furthermore the exact value $\sqrt{3}$ lies outside the estimated error margin of the result of the fit for $\lambda_{e}$.

Table IV lists the largest eigenvalues $\lambda_{o}^{(i, 5)}(p)$ of the majority-rule renormalization transformation matrix in the five-dimensional odd coupling subspace. A similar fit of the data yields an extrapolated eigenvalue $\lambda_{o}=2.80067(9)$, with $\chi^{2}=16$ which is to be compared with a number of 29 degrees of freedom. The statistical errors of the raw data are estimated as 0.00015 . The result for the leading irrelevant exponent is $y_{i}=-1.99(2)$. The exact value $3^{15 / 16}$ is also slightly outside the estimated uncertainty range about the extrapolated odd eigenvalue.
Next, we present the results of the modified rule. Table $\mathrm{V}$ lists the largest eigenvalues $\lambda_{e}^{(i, 5)}(p)$ of the modified rule renormalization transformation with $\omega=1.258$ in the fivedimensional even coupling subspace. These data show that the renormalization effect is much smaller than for the majority rule, which indicates that the fixed point has indeed moved towards nearest-neighbor Hamiltonian. A nonlinear least-squares fit yields the eigenvalue $\lambda_{e}$ as 1.7319(2), with $\chi^{2}=38$. The number of degrees of freedom is 29 in this fit. The statistical errors of the raw data are estimated as about 0.0003 . The result for $\lambda_{e}$ is much closer to the exact value than the result of the majority rule. Moreover, the exact value lies inside the error margin as produced by the fit. The result for the leading irrelevant exponent is $y_{i}=-1.2(1)$.

Table VI lists the eigenvalues $\lambda_{o}^{(i, 5)}(p)$ of the modified renormalization transformation with $\omega=1.258$ in the odd coupling space truncated to five dimensions. A similar fit yields the fixed-point eigenvalue $\lambda_{o}=2.80078(9)$, with $\chi^{2}$ $=10$. The number of degrees of freedom in this fit is 29 , and the statistical errors of the raw data are about 0.00015 . Again, the extrapolated value is much closer to the exact value than that of majority rule. The exact value lies just a little outside the $1 \sigma$ error margin of the least-squares fit. The result for the irrelevant exponent is $y_{i}=-1.6(1)$.

Some results for $y_{i}$ from the fits for $\lambda_{e}$ and $\lambda_{o}$ of the modified block-spin transformation are not close to the known Ising correction exponent -2. A somewhat speculative interpretation, mentioned in Sec. $\mathrm{V}$ involves the Potts subleading thermal exponent $-4 / 3[23]$. Setting $y_{i}=-4 / 3$, a

TABLE VI. Eigenvalues of the modified rule transformation matrix $(\omega=1.258)$ in the $n_{c}=5$ odd coupling subspace. The estimated statistical error in the data is approximately 0.00015 .

\begin{tabular}{cccccccc}
\hline \hline$i \backslash p$ & 10 & 9 & 8 & 7 & 6 & 5 & 4 \\
\hline 1 & 2.791732 & 2.791727 & 2.791731 & 2.791723 & 2.791697 & 2.791697 & 2.791701 \\
2 & 2.795678 & 2.795680 & 2.795682 & 2.795680 & 2.795620 & 2.795615 & 2.794304 \\
3 & 2.798618 & 2.798604 & 2.798606 & 2.798573 & 2.798551 & 2.797178 & \\
4 & 2.799832 & 2.799824 & 2.799811 & 2.799739 & 2.798382 & & \\
5 & 2.800394 & 2.800351 & 2.800366 & 2.798950 & & & \\
6 & 2.800635 & 2.800596 & 2.799194 & & & & \\
7 & 2.800731 & 2.799349 & & & & & \\
8 & 2.799424 & & & & & \\
\hline \hline
\end{tabular}


TABLE VII. Comparison of results of the majority rule and of the modified transformation. The exact values are $\lambda_{e}=1.73205 \ldots$ and $\lambda_{o}=2.80092 \ldots$

\begin{tabular}{cccc}
\hline \hline & Exact value & Majority rule & Modified rule \\
\hline$\lambda_{e}$ & $\sqrt{3}$ & $1.7330(2)$ & $1.7319(2)$ \\
$\lambda_{o}$ & $(\sqrt{3})^{15 / 8}$ & $2.80067(9)$ & $2.80078(9)$ \\
\hline \hline
\end{tabular}

fit of the data for $\lambda_{e}^{(i, 5)}(p)$ and $\lambda_{o}^{(i, 5)}(p)$ with expression (12) yields $\lambda_{e}=1.73177(8)$ and $\lambda_{o}=2.80109(8)$, respectively.

To compare the results of the two different block-spin transformations, we include the main results in Table VII. Apparently, the modified block-spin rule leads to a better estimation of leading eigenvalues of the linearized transformation matrix, both in the even and odd subspaces.

\section{B. Convergence with the dimensionality of the coupling subspace}

In the past years, the number of couplings $n_{c}$ used in MCRG analyses of the 3D Ising model has increased from 7 in Ref. [4] to 99 in Ref. [8]. The ordering of these couplings is a significant problem in order to avoid the situation that "important" couplings are left out, which would affect the resulting eigenvalue estimates, if not included in the analysis. An empirical criterion to distinguish "important" and "less important" couplings was introduced in Ref. [7]. This led to fast apparent convergence with increasing $n_{c}$ [7].

We have included up to ten even couplings in the analysis. They are shown in Fig. 2. We have performed fits of the largest eigenvalues according to Eq. (12) for $n_{c}=1$ to 10 even couplings. The results of the least-squares fits are shown in Fig. 4 for the majority rule and in Fig. 5 for the modified block-spin transformation with $\omega=1.258$. The eigenvalues display a satisfactory apparent convergence with the number of couplings. Statistically significant changes are absent for more than two couplings in the odd subspace and for more than five couplings in the even subspace. This holds for both types of block-spin transformation.

Thus we may be confident that the five-dimensional coupling subspace, used in the analysis presented in preceding section, is large enough.

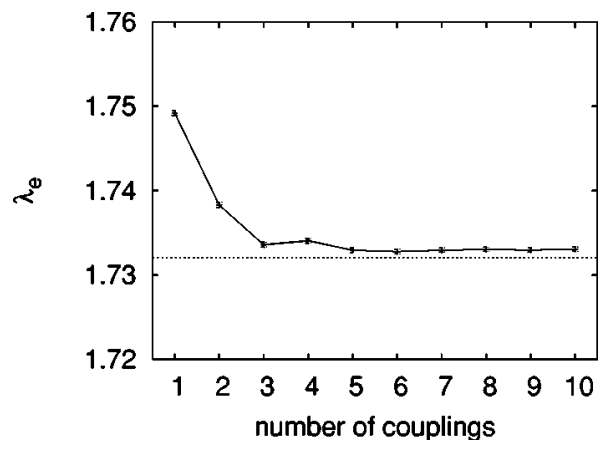

FIG. 4. Eigenvalues of the majority-rule transformation in the even subspace, extrapolated to the fixed point of the majority rule, as a function of the subspace dimensionality. The dashed line indicates the exact value $\sqrt{3}$.

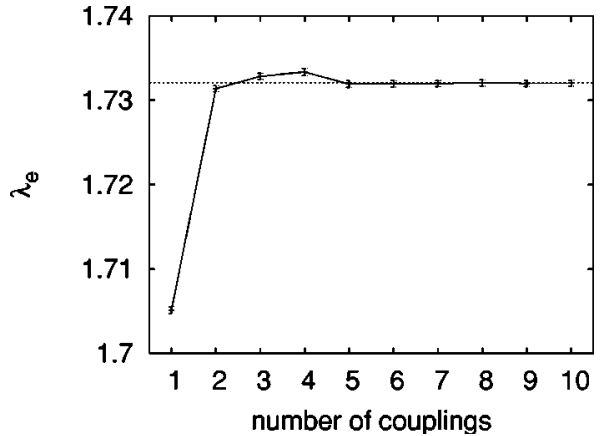

FIG. 5. Eigenvalues of the modified-rule transformation $(\omega$ $=1.258$ ) in the even subspace, extrapolated to the fixed point of the modified rule, as a function of the coupling subspace dimensionality. The dashed line indicates the exact value $\sqrt{3}$.

\section{DISCUSSION}

Our MCRG calculations on two-dimensional Ising model show that, just as for the three-dimensional model, the choice of a renormalization transformation with a fixed point with suppressed corrections to scaling leads to improved estimates of the renormalization exponents in comparison with the majority rule. Our choice for the transformation aims to put the fixed point on the nearest-neighbor Hamiltonian. This is convenient from a computational point of view [10] but the main reason behind this choice is that the leading correction to scaling vanishes in the nearest-neighbor triangular model. We have found that the majority rule has a fixed point with appreciable antiferromagnetic interactions with further neighbors. We have not explicitly calculated the corrections to scaling in this point. But the linear behavior of the transfer-matrix results presented in Fig. 3 indicates that the corrections are nonzero at the fixed point of the majority rule.

The failure of this fixed point to obey the analytic form of the renormalization equations is inconsistent with the prevailing description of the fixed point in the renormalization group theory. In order to fit such fixed points into the theory one has to abandon one or more of the aforementioned assumptions. Reference [24] uses a mechanism that explains the presence of corrections even at the fixed point and that generates nonanalytic contributions in the renormalized Hamiltonian. In the present work, we have successfully attempted to suppress the numerical problems such as associated with nonanalytic contributions by restoring consistency with the usual picture of vanishing correction amplitudes at the fixed point. Indeed we observed better convergence of the leading even and odd eigenvalues to their fixed-point values.

Another significant phenomenon is visible from the fixedpoint estimates in Tables I and II for the majority rule, which display a significant finite-size dependence, even up to relatively large sizes. This indicates that the assumption of locality of the renormalization transformation is not well satisfied. In comparison, the modified rule is better behaved: the value of $\omega$, which adjusts the fixed point to the nearest-neighbor Hamiltonian, has only a weak finite-size dependence. Furthermore, the estimated fixed point at the extrapolated value 
of $\omega$ displays a finite-size effect of only $10^{-3}$ for the smallest system, decreasing to $10^{-4}$ for the largest systems; this is small in comparison with the effects visible in Tables I and II.

Our analyses showed that the convergence to the fixed point, as deduced from the dependence of the largest eigenvalues on the number of renormalization steps, can be described by an effective exponent $y_{i}^{\prime}$ in the range $-2 \lesssim y_{i}^{\prime} \lesssim$ -1.2 , in some cases clearly different from the known irrelevant exponent -2 . Furthermore, the analysis of the secondlargest eigenvalue in the even subspace yields an irrelevant exponent $y_{i}=-1.62(5)$ for the majority rule and $y_{i}=$ $-1.26(5)$ for the modified rule. The error estimates in these two values are somewhat uncertain because of the occurrence of pairs of complex eigenvalues.

It is tempting to interpret this effective exponent in terms of two contributions: one with the expected irrelevant exponent -2 and another with an exponent $-4 / 3$ which is the subleading $q=2$ Potts temperature exponent [23]. However, for the $q$-state Potts model, the correction-to-scaling amplitudes associated with the subleading exponent seem to vanish just at $q=2$-i.e., the Ising model $[23,26]$. For $q=2$, the effects described by this exponent have been observed only in partial differential approximants [25] and in quantities involving explicit differentiations with respect to the number of states $q$ [26]. The theory of conformal invariance (see Ref. [27] and references therein) indeed predicts the existence of a set of exponents in the Ising model, including $-4 / 3$, that do not contribute to the thermodynamic properties. However, these exponents can still describe to other properties, such as percolative and geometric properties of Ising configurations [28]. Perhaps corrections with exponent $-4 / 3$ could enter in our analysis from nonthermodynamic geometric aspects introduced by the block-spin transformation.
The effect of the nonvanishing corrections to scaling at the fixed point of the majority-rule renormalization transformation, in terms of the deviations of the eigenvalues, seems less serious for the two-dimensional Ising model than for that in three dimensions [15]. This difference may be related to the values of the irrelevant exponents, which predict a faster decay of corrections in two dimensions.

As a concluding remark, we emphasize that, although our approach leads to a distinct improvement over the majority rule, the problem of the convergence to the fixed point is only partly solved; i.e., we still observe corrections that indicate that the fixed point does not yet precisely coincide with the simulated Hamiltonian. Further improvements may be achieved using block-spin transformations with more free parameters, so that the fixed point may be shifted to the nearest-neighbor Hamiltonian in a higher-dimensional coupling subspace.

\section{ACKNOWLEDGMENTS}

One of us (W.G.) wishes to thank the Abdus Salam ICTP, where part of this research was done, for hospitality. It is a pleasure to thank Professor J.M.J. van Leeuwen for a valuable discussion and X.F. Qian for contributing to the transfermatrix technique used to determine the correction-to-scaling amplitudes. This research is supported by the National Science Foundation of China under Grant No. 10105001, and by a grant from Beijing Normal University. It is also supported in part by the FOM ("Stichting voor Fundamenteel Onderzoek der Materie") which is financially supported by the NWO ("Nederlandse Organisatie voor Wetenschappelijk Onderzoek").
[1] S.-K. Ma, Phys. Rev. Lett. 37, 471 (1976).

[2] R. H. Swendsen, Phys. Rev. Lett. 42, 859 (1979).

[3] R. H. Swendsen, in Real Space Renormalization, edited by T. W. Burkhardt and J. M. J. van Leeuwen (Springer, Berlin, 1982).

[4] H. W. J. Blöte and R. H. Swendsen, Phys. Rev. B 20, 2077 (1979).

[5] G. S. Pawley, R. H. Swendsen, D. J. Wallace, and K. G. Wilson, Phys. Rev. B 29, 4030 (1984).

[6] R. Gupta, J. Appl. Phys. 61, 3605 (1987).

[7] H. W. J. Blöte, A. Compagner, J. H. Croockewit, Y. T. J. C. Fonk, J. R. Heringa, A. Hoogland, T. S. Smit, and A. L. van Willigen, Physica A 161, 1 (1989).

[8] C. F. Baillie, R. Gupta, K. A. Hawick, and G. S. Pawley, Phys. Rev. B 45, 10438 (1992).

[9] Y. Deng and H. W. J. Blöte, Phys. Rev. E 68, 036125 (2003).

[10] R. H. Swendsen, Phys. Rev. Lett. 52, 2321 (1984).

[11] F. J. Wegner, J. Phys. C 7, 2098 (1974).

[12] R. Shankar and R. Gupta, Phys. Rev. B 32, R6084 (1985).

[13] M. E. Fisher and M. Randeria, Phys. Rev. Lett. 56, 2332 (1986).

[14] A. C. D. van Enter, R. Fernández, and A. D. Sokal, J. Stat.
Phys. 72, 879 (1993).

[15] H. W. J. Blöte, J. R. Heringa, A. Hoogland, E. W. Meyer, and T. S. Smit, Phys. Rev. Lett. 76, 2613 (1996).

[16] Th. Niemeijer and J. M. J. van Leeuwen, Physica (Amsterdam) 71, 17 (1971); Phys. Rev. Lett. 31, 1411 (1973).

[17] U. Wolff, Phys. Lett. B 228, 379 (1989).

[18] Th. Niemeijer, and J. M. J. van Leeuwen, in Phase Transitions and Critical Phenomena, edited by C. Domb and M. S. Green (Academic, New York, 1971), Vol. 6.

[19] M. P. Nightingale, Phys. Lett. 59, 486 (1977); Proc. K. Ned. Akad. Wet., Ser. B: Palaeontol., Geol., Phys., Chem., Anthropol. 82, 235 (1979)

[20] H. J. Hilhorst, M. Schick, and J. M. J. van Leeuwen, Phys. Rev. B 19, 2749 (1979).

[22] M. R. Dudek, J. M. J. van Leeuwen, and H. W. J. Blöte, Physica A 147, 344 (1987).

[23] B. Nienhuis, in Phase Transitions and Critical Phenomena., edited by C. Domb and J. L. Lebowitz (Academic, London, 1987), Vol. 11.

[24] R. H. Swendsen, in Computer Simulation Studies in Condensed Matter Physics, edited by D. P. Landau, S. P. Lewis, and H. B. Schüttler (Springer-Verlag, Berlin, 2002). 
[25] M. Barma and M. E. Fisher, Phys. Rev. Lett. 53, 1935 (1984); Phys. Rev. B 31, 5954 (1985).

[26] H. W. J. Blöte and M. P. M. den Nijs, Phys. Rev. B 37, 1766 (1988).

[27] J. L. Cardy, in Phase Transitions and Critical Phenomena, edited by C. Domb and J. L. Lebowitz (Academic, London, 1987), Vol. 11.

[28] H. W. J. Blöte, Y. M. M. Knops, and B. Nienhuis, Phys. Rev. Lett. 68, 3440 (1992). 